\title{
An investigation of musical timbre
}

\author{
S.N. MALLOCH and A.M. CAMPBELL
}

Room 4214, JCMB Physics Department, The King's Buildings, University of Edinburgh, Mayfield Road, Edinburgh EH9 3JZ, U.K.

\begin{abstract}
Musical timbre is a particularly difficult attribute to measure and quantify. However, Pollard and Janson (1982) [3] have successfully plotted the changing timbre of the starting transient and steady state of a single note. Following on from their work, the changing timbre of an ensemble piece of music is graphed using just two independent parameters, these being derived from Stevens (1971) [4], Mark VII.
\end{abstract}

\begin{abstract}
1. Introduction
When faced with the need to discuss the timbre of a piece of music, the analyst of a piece of music's form and structure is severely restricted by the lack of a specific language with which to describe what is heard. The repercussions of this analytical gap vary in importance depending on the genre of music under consideration. For a piece which does not appear to use timbre as an important means of articulating structure, the absence of an analytical timbral language may not be a significant encumbrance to an understanding of the overall structure of the piece. However, consider the effect of playing in piano reduction a 20 th century orchestral work such as Xenakis' Pithoprakta, or pieces by Ligeti or Lutoslawski. The basic nature of the piece would be grossly distorted. With this sort of music, timbre is not just a carrier for more structurally important information, it is vital for our understanding of the music. In order to discuss this type of music, a more precise analytical language for music timbre would be helpful.
\end{abstract}

2. Theoretical Background

The timbre of a musical sound depends on our perception of that sound's component sirusoids - their amplitudes, the way they change through time, the way they are spaced, and, of lesser importance, their phase. Futting the phase information to one side, a spectrograph is a very useful tool for examining the sinusoidal makeup of a sound (see Figure 1). This spectrograph was produced by the software Hypersignal ${ }^{1}$, using data sampled from a Compact Disk at $44.1 \mathrm{kHz}$, then subjected to an FFT of order 12, framesize of 4096 samples, overlap of 2048, and using a Hamming Window.

The information on our perception of timbre is enbedded in this spectrographic data. Robert Cogan (1984) [1] attempted to extract timbre information visualiy from the raw data of a spectrograph. This method may be assisted by applying a 
perceptual weighting factor to the data. Figure 2 represents the data of Figure 1 after an A-weighting has been applied.

While Figure 2 is closer to a representation of the way we perceive the music than Figure 1, it still does not get us closer to extracting specifically timbre information. In addition, the weighting factor does not take into account the more subtle perceptual effects as expressed in the set of equal-loudness contours, nor does it address the effects of masking.

3. Timbre Data Extraction and Reduction

John Grey (1975) [2] found that three dimensions were sufficient to plot the perceptual data obtained by asking people to discriminate between 16 instrumental tores, equalised for pitch, duration and loudness. Taking their cue from this number of dimensions, Pollard \& Janson (1982) [3] have proposed a method of graphing the timbre of a single note, which normalises data for pitch and loudness. 1 Three measurements are extracted from spectrographic data and used to plot a single point on a triangular graph, named a tristimulus diagram. A point on the graph represents the timbre of a note at a particular instant of time.

\subsection{Method}

By adapting Pollard and Janson's work, a representation of the instantaneous timbre of a piece of ensemble music may be obtained by extracting just three values.

A spectrograph is produced of un-weighted data, an example of which is Figure 1. This data is then subjected to the process of calculating a total loudness in sones for a particular time instant, as presented by Stevens (1971) [4] in his method known as Mark VII. This method divides the sound into one-third octave bands, then calculates the total loudness by finding the loudest band and applying variables from look-up tables which allow interband masking and the non-linear response of the ear to be taken into account.

Once the calculations of Stevens' Mark VII are complete, three values are extracted. Orne is the loudness of the loudest one-third octave band averaged over a time interval (determined by the software as an interval of $45 \mathrm{~ms}$ ) $-L_{1}$. The other two consist of a loudness values for all sound energy above this band $\left(L_{a}\right)$ and a value for all sound energy below $\left(L_{b}\right)$. These values are then taken as fractions of the total loudness (Lt):

$L_{t}=L_{1}+L_{a}+L_{b}$

where $L$ is loudress in sones.

Three values may then be derived:

$x=L_{a} / L_{t}$
$y=L_{b} / L_{t}$
$z=L_{1} / L_{t}$

As $x+y+z$ must equal 1 , only two values need to be plotted.

1. It is understood that components in a sound other than those utilised in the tristimulus diagram and discussed later in this paper, will play a role in timbre perception. However, the acousic components of timbre discussed here are considered to be primary to our perception. 
The graph of values $x$ and $y$, derived from the data represented in Figure 1 , is shown in Figure 3. The higher the value for $x$ or $y$, the greater the loudness of the sound energy above or below the loudest band, relative to the total loudness. The closer the traces for $x$ and $y$ are to each other, the greater the amount of sound energy concentrated in the loudest band, relative to the total loudness. These relationships play a highly important role in our experience of a piece's timbre.

\section{Conclusions}

Pollard and Janson [3] have produced an effective means of plotting the changing timbre of a single note. Presented here is a means of adapting their methodology so that entire pieces of ensemble music may be analysed, and thus a measure of the changing timbre produced. These measurements, combined with information on harmonicity and acoustic dissonance will produce an even fuller account of how we perceive the sound of a piece of music.

\section{References:}

[1] Cogan R., New Images of Musical Sound (Harvard University Press, Cambridge, Mass., 1984).

[2] Grey J., An Exploration of Musical Timbre using computer based techniques for analysis, synthesis and perceptual scaling. Ph.D. dissertation, Stanford University, 1975.

[3] Pollard $H$. and Janson E., A Tristimulus Method for the Specification of Musical Timbre. Acustica 51 (1982) 162-171.

[4] Stevens S., Perceived Level of Noise by Mark VII and Decibels (E). The Journal of the Acoustical Society of America 51 (1972) 575-601.

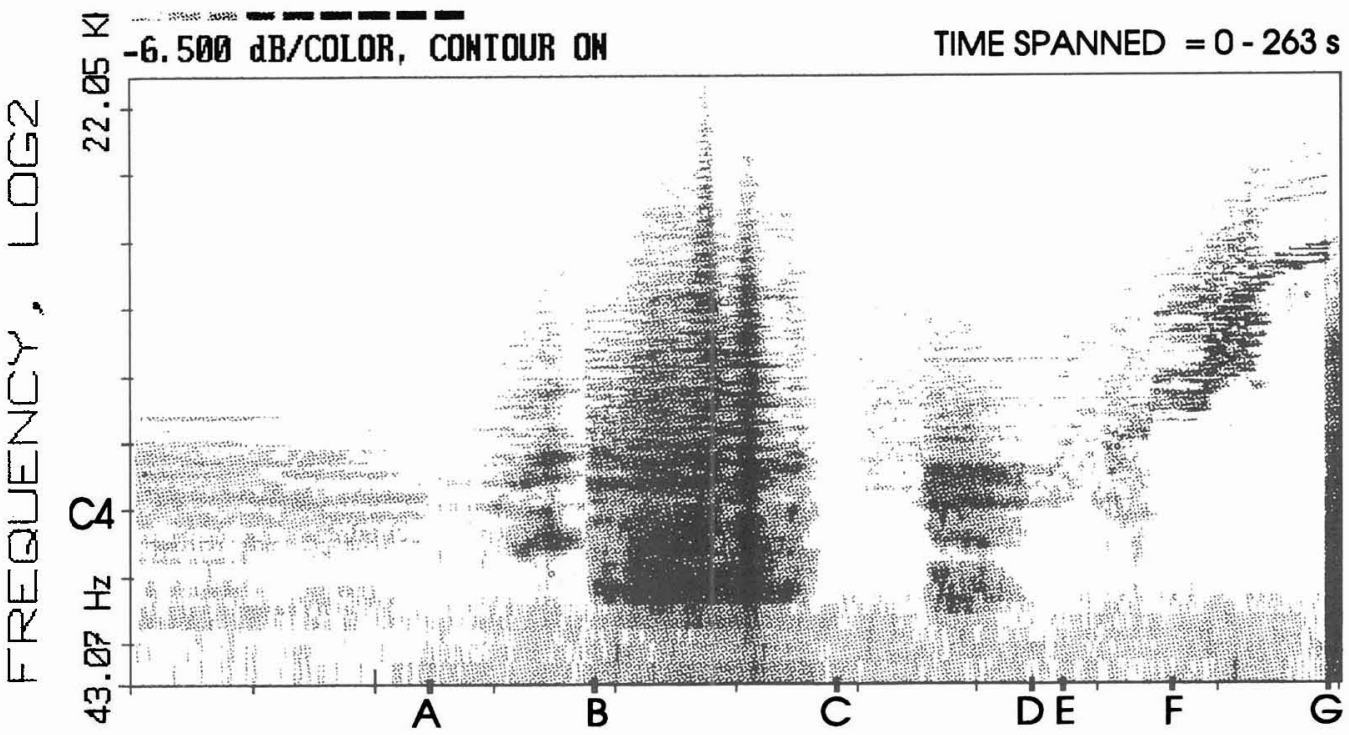

Figure 1. A spectrograph of the first half of Ligeti's Atmospheres (1961), for large orchestra. Letters along the time axis are rehearsal letters from the score. The position of the pitch C4 is noted on the frequency axis. 


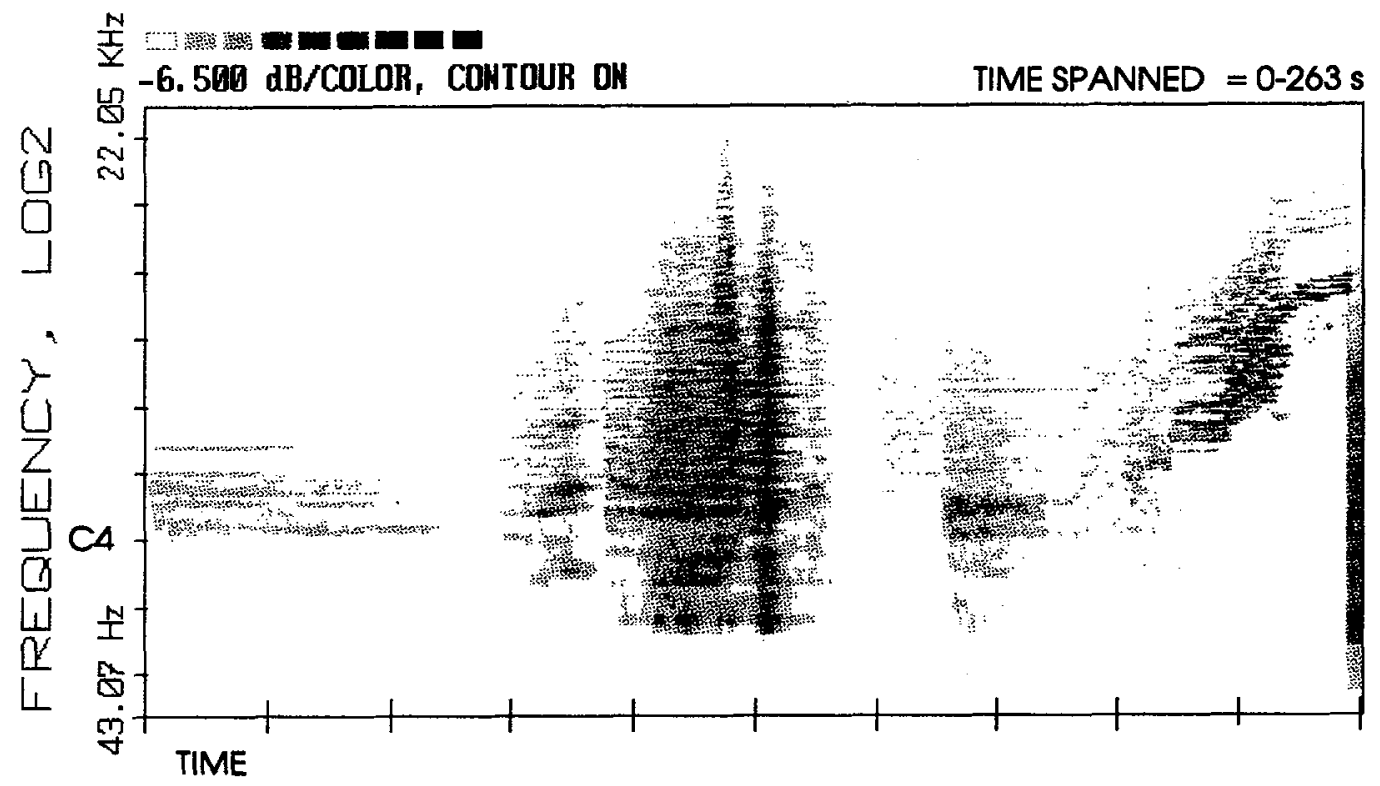

Figure 2. An Aweighted version of the data shown in Figure 1.

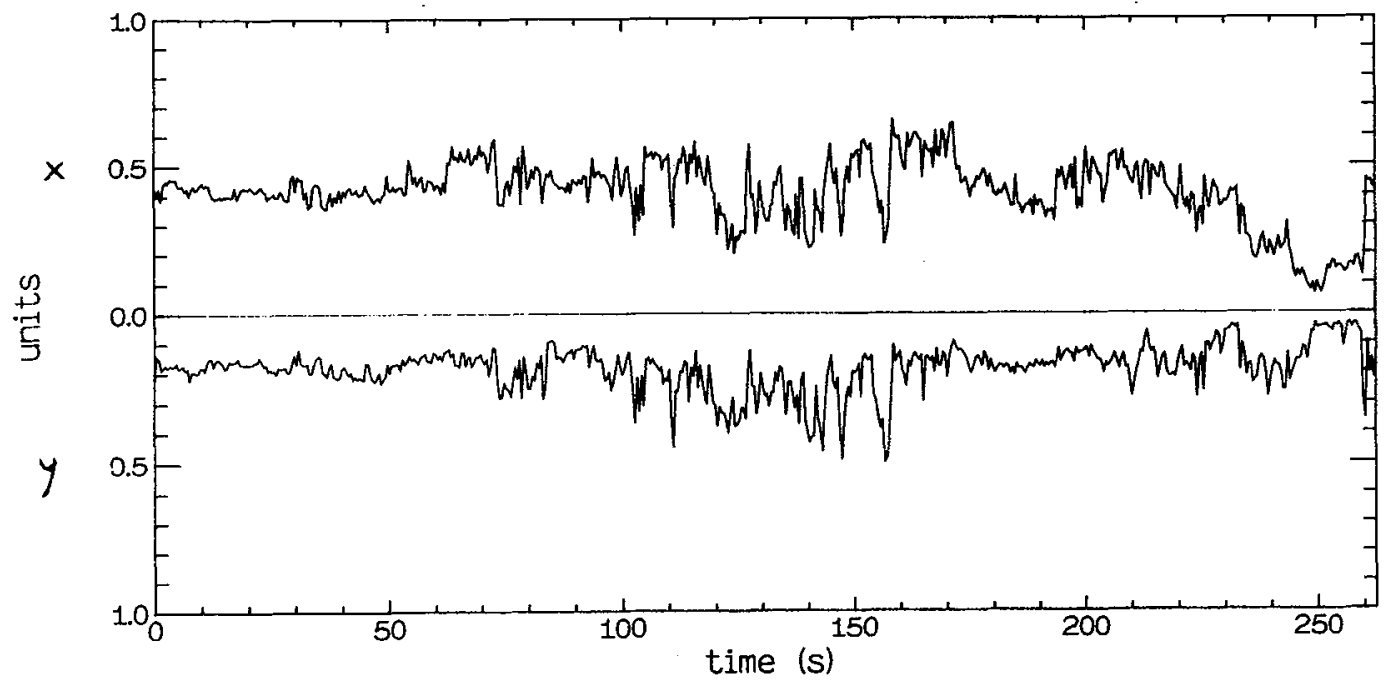

Figure 3. A graph of $x$ and $y$ against time, derived from the data shown in Figure 1. 